\title{
Preparation of Hollow-Porous Rosin-Based Polyurethane Microspheres with pH-Responsive Characteristics
}

\author{
Caili Yu, Hao Liu, Jintao Shao and Faai Zhang \\ College of Chemistry and Biology Engineering, Guilin University of Technology, Guilin, 541004, China \\ ${ }^{*}$ Corresponding Author: Faai Zhang. Email: zhangfaai@glut.edu.cn
}

Received: 01 May 2021 Accepted: 02 July 2021

\begin{abstract}
Preparation of polymer microspheres from naturally occurring resource is a challenge. Here, a rosin-based polyol (RAG) was used to prepare polyurethane resin (RPU) firstly, and then act as both self-assembled precursor and emulsifier, rosin based polyurethane microspheres (RPUMs) were prepared. In the process of self-emulsification, the RPU formed vesicles by self-assembly. The outer shell of the vesicle consisted of hydrophilic segments, while the inner shell contained the hydrophobic phase. After cross-linking the RPU and removal of the solvent in the core, the porous-hollow microspheres with $\mathrm{pH}$-sensitive were obtained. The microspheres were characterized by optical microscope (OM), scanning electron microscopy (SEM) and transmission electron microscope (TEM). The effect of type and amount of the hydrophilic chain extender, and solvent on the morphology, particle size and distribution, and buffer volume of the microspheres were determined. The best conditions for synthetic RPUMs were as follows: $n_{\mathrm{NCO}} / n_{\mathrm{OH}}=1, n_{\mathrm{RAG}}: n_{1 \text {-(2-hydroxyethyl) piperazine }}=4: 6$, with azodiisobutyronitrile level of 1.0 wt.\%, based on reactive monomers, mixing speed of both emulsification and polymerization at $400 \mathrm{r} \cdot \mathrm{min}^{-1}$, the RPUMs synthesized had porous-hollow structure with a buffer volume of $1.6 \mathrm{~mL}$.
\end{abstract}

\section{KEYWORDS}

Polyurethane microspheres; rosin-based; pH-responsive; hollow-porous

\section{Introduction}

In recent years, research on green renewable energy has become a global hot spot due to fossil fuel depletion and environmental pollution [1]. As a kind of renewable resources with great potential application, biomass is utilized to synthesize high value-added chemicals, making it as one of the important means to solve the global energy crisis [2]. Rosin is one of the most commonly used biomass with the advantages of abundance, low price, and easy modification. In addition, the characteristic hydrophenanthrene ring structure of rosin is similar to some cycloaliphatic and aromatic compounds, making its derivatives as potential substitutes for fossil-based compounds [3]. Unfortunately, up to date, researches of using rosin as raw material for preparation of valuable chemicals are generally unsatisfactory. There is a burning need to broaden its application field.

Polyurethanes (PUs) have been widely used in rigid foams [4], elastomers [5], adhesive [6], coatings [7] and biomaterials [8] due to their unique performance and various morphological structures. Among these morphologies studied, porous-hollow PU microspheres have recently aroused considerable interests. 
Previous studies has proved that porous-hollow microcapsules or microspheres show great potential for applications in the fields of adsorbents [9], tissues engineering [10], drug diagnosis [11], environmental protection [12], etc., owing to their high specific area, good flow ability and surface permeability. In current practice, electrospraying [13] and template-assisted technique [14] are main methods to synthesize porous-hollow PU microspheres. However, these methods require multi-step preparation procedures. Therefore, developing a novel and facile method to prepare porous-hollow PU microspheres are highly desired.

Intelligent materials perform self-regulating behavior under the stimulation of $\mathrm{pH}[15,16]$, temperature [17], light [18], etc., have drawn much attention in material science, tissue engineering and drug delivery systems [19]. It is well known that different parts of the human body have different $\mathrm{pH}$ values [20]. Thus, $\mathrm{pH}$-sensitive materials can adjust their structure and conformation by ionizing functional groups through the process of ionization and deionization. For example, tumors or inflammation areas in the human body are often accompanied by acidosis, which could be employed as a target for $\mathrm{pH}$-sensitive administration [21].

The development of intelligent materials has become the focus in the world. In order to widen the application field of RPU and improve the added value of rosin, a new type of functional PU based on rosin was developed. Then, rosin-based PU microspheres (RPUMs) are prepared using the RPU as both self-assembled precursor and emulsifier. More precisely, in the process of self-emulsification, the RPU forms vesicles by self-assembly. The outer shell of the vesicle consists of the ordered arrangement of hydrophilic segments, while the inner shell consists of the hydrophobic phase (e.g., hydrophobic segments, inert solvent). After cross-linking the RPU and removal of the solvent in the core, the porous-hollow microspheres with $\mathrm{pH}$-sensitive are obtained.

\section{Materials and Methods}

\subsection{Materials}

An ester of acrylic rosin and glycidyl methacrylate (RAG, 97\%, acid value: $4 \mathrm{mgKOH} / \mathrm{g}$, hydroxyl value: $170 \mathrm{mgKOH} / \mathrm{g}$ ) was made by our laboratory according to literature [12]. Hexamethylene diisocyanate (HDI, 99\%) and methyldiethanolamine (MDEA, 99\%) were purchased from Aladdin industrial corporation. Acetone, hydrochloric acid, sodium hydroxide, di-n-butylamine, dibutyltin dilaurate (DBTDL) and azodiisobutyronitrile (AIBN) were purchased from Xilong Scientific Co. (Shantou, China) Butyldiethanolamine (BDEA, 98\%), bis-1, 4-(2-hydroxyethyl) piperazine (BHEP, 98\%), 1-(2-hydroxyethyl) piperazine (HEP, 98\%), carbon tetrachloride $\left(\mathrm{CCl}_{4}\right)$ and butyl alcohol (NBA) were purchased from Sinopharm Chemical Reagent Co., Ltd. (Shanghai, China) Toluene and N, Ndimthylformamide (DMF) were obtained from Guangdong Xilong Chemical Co., Ltd., Shantou, China.

\subsection{Methods}

The RAG (in toluene) and DBTDL ( $0.5 \mathrm{wt} . \%$ of reactive monomer) were mixed firstly. A three-necked flask containing the reaction mixture, fitted with a stirrer, a reflux condenser, and a nitrogen inlet, was heated to $55^{\circ} \mathrm{C}$. Next, HDI was introduced into the system for the reaction. When RAG reacted with HDI for $2 \mathrm{~h}$, the-NCO content was reduced to the theoretical value ( $\sim 3 \%$, according to acetone-di-n-butylamine method), a chain extender $\left(n_{\mathrm{RAG}+\text { chain extender }}: n_{\mathrm{HDI}}=1: 1\right)$ was added. When the-NCO content dropped to zero, the system was cooled down to $25^{\circ} \mathrm{C}$ with ice water. Subsequently, acetic acid was added as a neutralizer under stirring for $15 \mathrm{~min}$. The rosin-based PU (RPU) in toluene was obtained.

At room temperature, a designed amount of RPU solution and an initiator AIBN ( $1 \mathrm{wt} \%$ of reactive monomer) were mixed uniformly using ultrasonicator. Then, the mixed solution was added to a threenecked flask containing $60 \mathrm{~mL}$ of distilled water and heated to $80^{\circ} \mathrm{C}$ under $\mathrm{N}_{2}$ protection for $3 \mathrm{~h}$. After centrifugation the mixture at $3000 \mathrm{r} \cdot \mathrm{min}^{-1}$ for $15 \mathrm{~min}$, washing with distilled water, secondary centrifugation and vacuum drying, the RPUMs were obtained. 
Fourier transform infrared (FTIR) spectrum of RPUMs was recorded on an American Thermo scientific IS10 Fourier Transform Infrared Spectrometer in the range of $400-4,000 \mathrm{~cm}^{-1}$. After vacuum drying, the samples were mixed with $\mathrm{KBr}$ and tabletted. The morphologies of the RPUMs were observed with optical microscope (OM) (DMXRP, Germany). The surface morphology of RPUMs (80 260 mesh) was analyzed by using Japanese Electron Company JSM-6380LV scanning electron microscope. The internal structure of the microspheres (80 260 mesh) was observed by Japanese high-Tech Company JEM-2100F field emission transmission electron microscope.

The particle diameters of RPUMs were determined by image analysis using Nano measurer (DMXRP, Germany), which selected 100 microspheres from optical microscope photos. The particle size and particle distribution index (PDI) of the microspheres were calculated as follows:

$D_{n}=\sum_{i=1}^{n} d_{i} / n$

$D_{w}=\sum_{i=1}^{n} d_{i}^{4} / \sum_{i}^{n} d_{i}^{3}$

$P D I=D_{w} / D_{n}$

where $D_{\mathrm{n}}$ is the number average particle size $/ \mu \mathrm{m} ; D_{\mathrm{w}}$ is the weight average particle size/ $\mu \mathrm{m} ; n$ is the number of microspheres measured.

The $\mathrm{pH}$ response behavior of the microspheres was determined by acid-base titration. RPUMs suspension in water $(2 \mathrm{mg} / \mathrm{mL})$ was prepared after adjusting $\mathrm{pH}=2$ by $\mathrm{HCl}$ and stirring for $30 \mathrm{~h}$. Titrations were carried out by stepwise addition of $500 \mu \mathrm{L}$ of $\mathrm{NaOH}(0.05 \mathrm{~mol} / \mathrm{L})$ with $3 \mathrm{~min}$ interval for equilibrium each time. The $\mathrm{pH}$ value was checked by a $\mathrm{pH}$ meter (PHS Type, Shanghai Electronic Science Instrument Co., Shanghai, China). The buffer capacity of RPUMs was calculated from the titration curves. The ionization degree $(\alpha)$ of the amino groups in the RPUMs was calculated through Eqs. (4) and (5) [22].

$\Delta V_{\mathrm{NaOH}}=V_{2}-V_{1}$

$\alpha=\frac{[\text { basic }]-\left[\mathrm{OH}^{-}\right]+\left[\mathrm{H}^{+}\right]}{C_{N H}}$

where $V_{1}$ is the $\mathrm{NaOH}$ consumption volume corresponding to the first mutation point, $V_{2}$ is the $\mathrm{NaOH}$ consumption volume corresponding to the second mutation point; $[$ basic $],\left[\mathrm{OH}^{-}\right]$and $\left[\mathrm{H}^{+}\right]$are the molar concentrations of the added $\mathrm{NaOH}$ for titration, free hydroxide ion and hydrogen ion, respectively, and $C_{\mathrm{NH}}$ is the total molar concentration of the amino groups in RPUMs. The $\mathrm{pH}$ value at $\alpha=0.5$ is considered as the apparent dissociation constant $\mathrm{pK}_{\mathrm{a}}$ of PUs.

\section{Results and Discussion}

\subsection{Effect of Hydrophilic Chain Extenders on the Properties of Microspheres}

When the porogen was fixed as toluene, with the solid content of RPU (in toluene solution) of $25 \%$, $n_{\mathrm{RAG}}: n_{\text {chain extender }}=4: 6$, stirring speed for both polymerization and emulsification were $400 \mathrm{r} \cdot \mathrm{min}^{-1}$ and reaction temperature at $80^{\circ} \mathrm{C}$, different types of hydrophilic chain extender (Fig. 1) were used to prepare the RPUMs, and their effects on the particle size, PDI, and dissociative parameters of the RPUMs are shown in Tables 1 and 2, the optical microscope photographs and $\mathrm{pH}$ sensitivity of the RPUMs are presented in Figs. 2 and 3, respectively. 


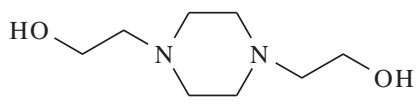

BHEP<smiles>CN(CCO)CCO</smiles>

MDEA

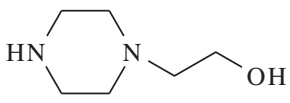

HEP

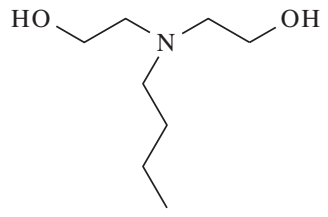

BDEA

Figure 1: Molecular formula of hydrophilic chain extender

Table 1: Effect of different chain extender on the RPUMs

\begin{tabular}{lllll}
\hline Hydrophilic chain extender & $\mathrm{D}_{\mathrm{n}} / \mu \mathrm{m}$ & $\mathrm{D}_{w} / \mu \mathrm{m}$ & PDI & Yield $\%$ \\
\hline HEP & 25.1 & 30.8 & 1.22 & 92.4 \\
BHEP & - & - & - & 88.7 \\
MDEA & 54.8 & 74.3 & 1.36 & 89.3 \\
BDEA & 72.3 & 87.3 & 1.21 & 91.1 \\
\hline
\end{tabular}

Table 2: Dissociative parameters of the RPUMs with different chain extender

\begin{tabular}{lllll}
\hline Hydrophilic chain extender & BHEP & HEP & MDEA & BDEA \\
\hline $\mathrm{pK}_{\mathrm{a}}$ & 6.65 & 6.47 & 6.45 & 6.28 \\
$\Delta \mathrm{V}_{\mathrm{NaOH}} / \mathrm{mL}$ & $1.6 \pm 0.01$ & $1.5 \pm 0.03$ & $2.6 \pm 0.03$ & $2.5 \pm 0.01$ \\
$\alpha$ & 0.4890 & 0.4934 & 0.5597 & 0.5875 \\
\hline
\end{tabular}

It can be seen from Fig. 2 and Table 1 that the average particle sizes of the RPUMs are large when the hydrophilic chain extenders are MDEA and BDEA, and some of the microspheres are broken to certain degrees. However, the microspheres from BHEP as hydrophilic chain extender are not spherical and broken into irregular blocks. The main reason is that the oil-in-water emulsion formed by selfemulsification is based on hydrophilic segment in RPU as outer shell, and hydrophobic segment as inner sphere shell, toluene as core. The size of the emulsion is positively related to the self-emulsifying ability of the RPU. When the RPU is neutralized by acetic acid, the tertiary amine groups in the chain combine $\mathrm{H}^{+}$with the ionized $\mathrm{CH}_{3} \mathrm{COO}^{-}$to form a double electric layer. The stronger the ability of tertiary amine group to bind $\mathrm{H}^{+}$, the higher the concentration of ionized $\mathrm{CH}_{3} \mathrm{COO}^{-}$, the thicker the electric double layer, the stronger the hydrophilic ability of RPU. HEP and BHEP have stronger hydrophilic ability than MEDA and BDEA. In the case, the resistance of self-emulsifying and phase inversion is small, thus the oil phase is easier to be dispersed in the water phase, smaller particle size formed [23].

In addition, the molecular structures of MDEA and BDEA are flexible chains with strong molecular mobility. During the polymerization process, toluene continuously volatilizes out of the shell to form a cavity. At the same time, due to the combined action of collision between latex particles, it is difficult to maintain the shape of the shell layer of the microspheres with insufficient rigidity, resulting in depression 
and collapse of the microspheres. HEP contains six-membered alicyclic structure, compared with the chain structure of MDEA and BDEA, it has stronger rigidity and can confine the thermodynamic movement of the chain segment. In the process of spheroidization, HEP can alleviate the shell of microspheres from depressing or broken due to solvent volatilization and collision between microspheres, and maintain relatively good spherical shape. The molecular structure of BHEP is highly symmetrical, with good regularity of hard segment, strong rigidity, and difficult to be emulsified into spheres. Among the four types of hydrophilic monomers, the protonation ability of BHEP per unit volume is the strongest, and it is easy to be swelled after the formation of microspheres. With the further volatilization and diffusion of toluene from the spherical core, the cavity expands further and the swelling effect intensifies, so it is difficult to get a better spherical shape.

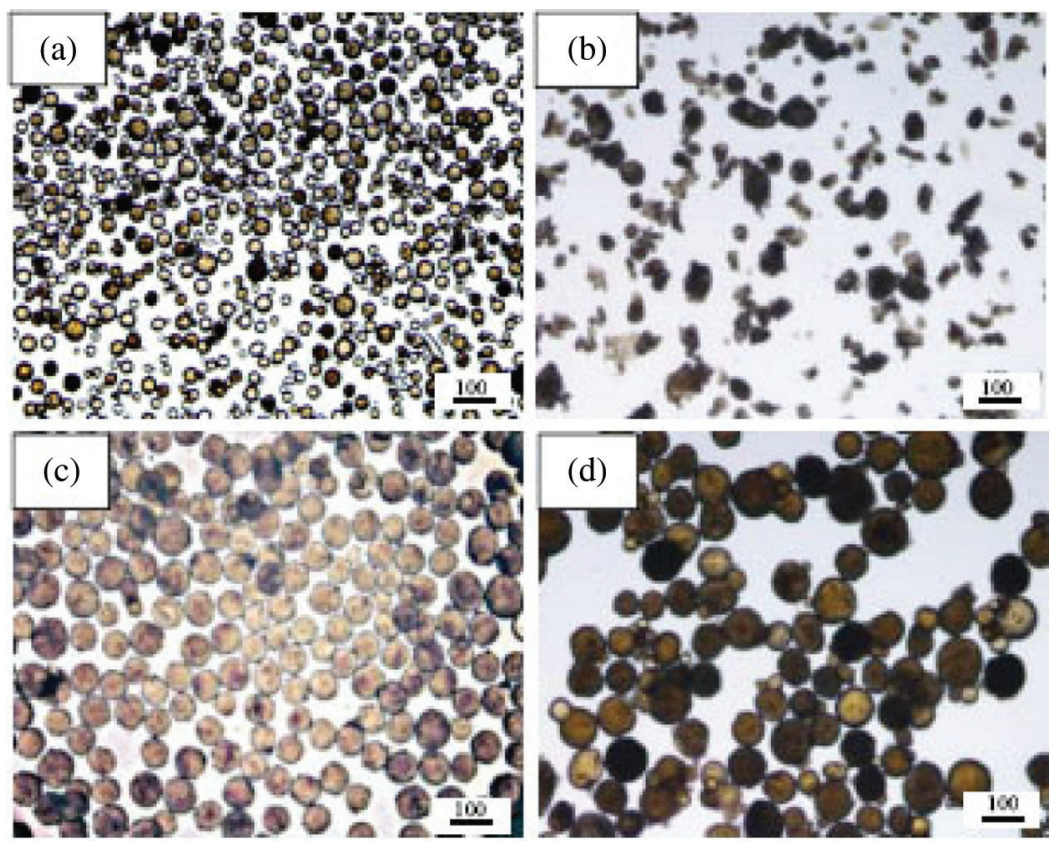

Figure 2: Optical microscope photos of the RPUMs with different hydrophilic chain extender (a: HEP; b: BHEP; c: MDEA; d: BDEA)

As can be seen from Fig. 3 and Table 2 that there are buffer platforms near $\mathrm{pH}=6 \sim 7$ for the microspheres with four chain extenders, and the dissociation constant $\mathrm{pK}_{\mathrm{a}}$ from large to small is BHEP, HEP, MDEA, BDEA, and the dissociation degree $\alpha$ for MDEA and BDEA is higher than that of HEP and BHEP. From the molecular structure of hydrophilic chain extender in Fig. 1, there are two tertiary amine groups in the structure of BHEP, and one tertiary amine group and one secondary amine group for HEP, and only one tertiary amine group in MDEA and BDEA. The longer -N side chain in BDEA lead to higher electron cloud density and stronger electron donating effect. The tertiary amine shows weaker binding ability to $\mathrm{H}^{+}$, and forms unstable $-\mathrm{NH}^{+}$. The stronger the binding ability of hydrophilic chain extender to $\mathrm{H}^{+}$, the weaker the acidity of $-\mathrm{NH}^{+}$as proton donor, the lower the $\mathrm{K}_{\mathrm{a}}$ and the higher the $\mathrm{pK}_{\mathrm{a}}$.

It can be seen from Table 2 that the $\alpha$ value of HEP is higher than that of BHEP. Although their molecular structures are similar, the urea group is formed after HEP is introduced into the PU chain segment. In the case, the electron cloud on the tertiary amine group in the urea group is shifted due to the electronwithdrawing effect of carbonyl group and $\mathrm{p}-\pi$ conjugation effect, so it is not easy to be combined with the empty orbit on $\mathrm{H}^{+}$to produce protonation, and $-\mathrm{NH}^{+}$combined with $\mathrm{H}^{+}$is more prone to produce 
deprotonation effect. The dissociation ability and dissociation degree of $-\mathrm{NH}^{+}$in $\mathrm{RPUMs}$ are enhanced, but the buffer volume of RPUMs with HEP is smaller than that of BHEP. Similarly, MDEA and BDEA have stronger deprotonation ability, and $-\mathrm{NH}^{+}$obtained by combining tertiary amine with $\mathrm{H}^{+}$has stronger dissociation ability. Therefore, the concentration of tertiary amine in RPUMs is low, and the final dissociation degree increases. Considering their small sizes, high strengths, and buffering capacities of the microspheres, HEP was selected as the chain extender for the following experiments.

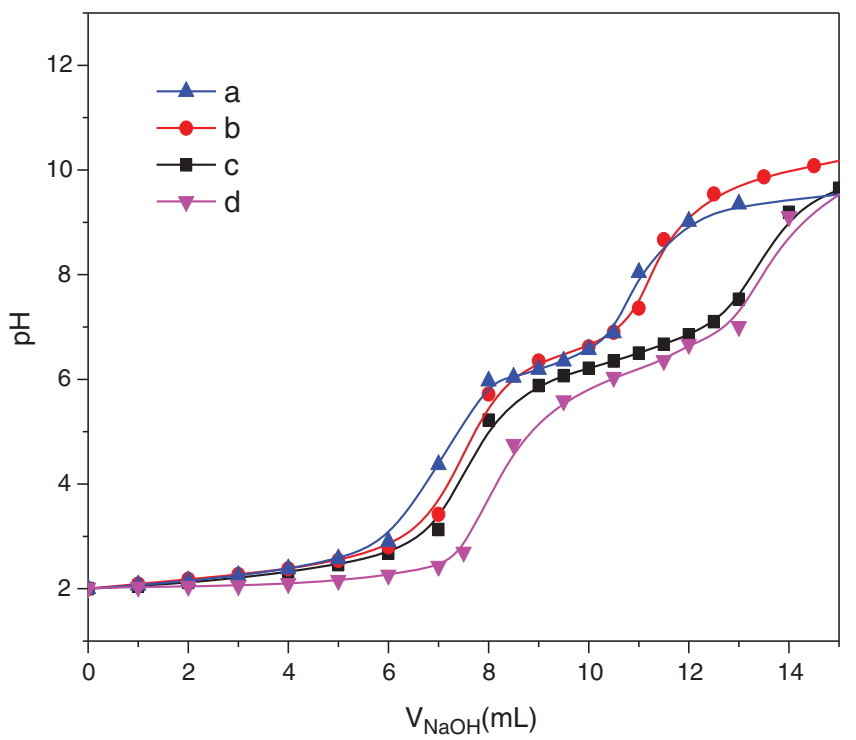

Figure 3: Effect of different hydrophilic chain extender on the pH sensitivity of the RPUMs (a: HEP; b: BHEP; c: MDEA; d: BDEA)

\subsection{Effect of Hydrophilic Chain Extender Amount on Properties of Microspheres}

At the solid content of RPU solution of $25 \%$, the effects of hydrophilic chain extender ratios, $n_{\mathrm{RAG}} / n_{\mathrm{HEP}}$ on the performance of RPUMs were studied, and the results are shown in Tables 3 and 4, Figs. 4 and 5. It can be seen from Table 3 and Fig. 4 that RPUMs have a better spherical shape. As the amount of hydrophilic chain extender HEP decreases, the particle size of the obtained microspheres increases with less regular spherical shape and rough surface of the microspheres, and the yield gradually decreases. As the proportion of hydrophilic segments in the RPU molecule decreases, the resistance to the formation of the oil-in-water emulsion increases, and the particle size of the latex particles gradually increases.

Table 3: Effects of hydrophilic chain extender amounts on RPUMs

\begin{tabular}{lllll}
\hline$n_{\text {RAG: }} n_{\text {HEP }}$ & $\mathrm{D}_{\mathrm{n}} / \mu \mathrm{m}$ & $\mathrm{D}_{w} / \mu \mathrm{m}$ & PDI & Yield/\% \\
\hline $4: 6$ & 25.1 & 30.8 & 1.22 & 92.4 \\
$5: 5$ & 62.6 & 77.6 & 1.24 & 91.8 \\
$6: 4$ & 97.3 & 125.5 & 1.29 & 90.4 \\
$7: 3$ & 132.9 & 154.9 & 1.17 & 88.2 \\
\hline
\end{tabular}




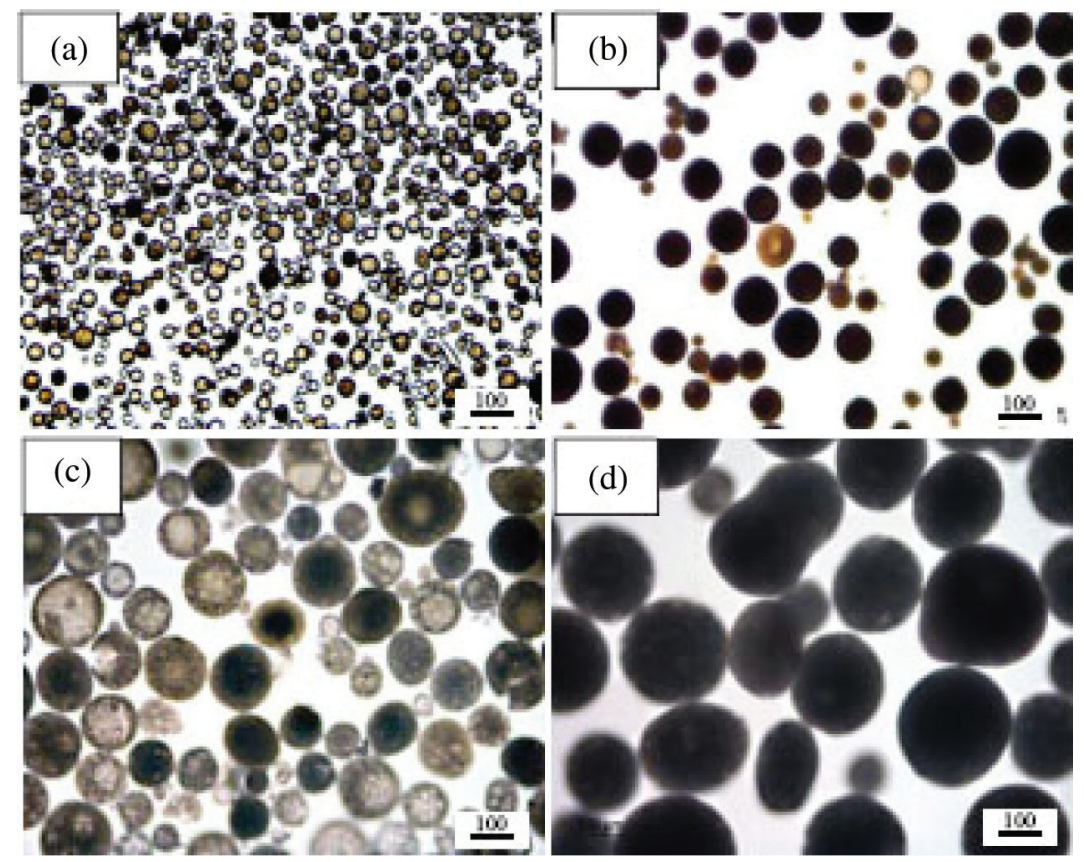

Figure 4: Optical microscope of RPUMs with different amounts of hydrophilic chain extender ( $n_{\text {RAG }}: n$ HEP, a: 4:6; b: 5:5; c: 6:4; d: 7:3)

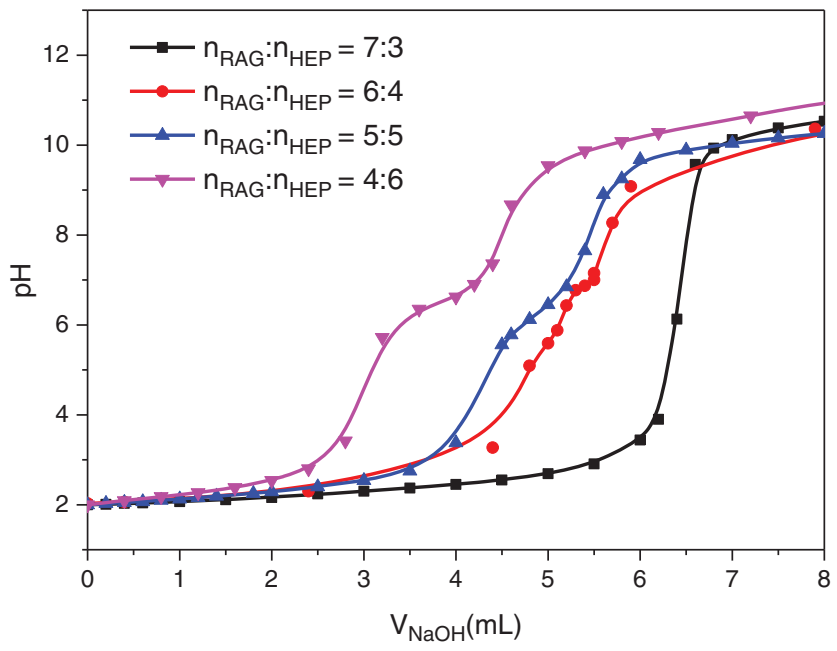

Figure 5: Effect of different amounts of hydrophilic chain extender on the $\mathrm{pH}$ sensitivity of RPUMs

Table 4: Dissociative parameters of RPUMs with different amounts of chain extender

\begin{tabular}{lllll}
\hline$n_{\mathrm{RAG}:} n_{\mathrm{HEP}}$ & $4: 6$ & $5: 5$ & $6: 4$ & $7: 3$ \\
\hline$\Delta \mathrm{V}_{\mathrm{NaOH}} / \mathrm{mL}$ & $1.5 \pm 0.03$ & $0.8 \pm 0.03$ & $0.6 \pm 0.03$ & - \\
$\alpha$ & 0.4934 & 0.6739 & 0.7032 & - \\
\hline
\end{tabular}


As can be seen from Fig. 5 and Table 4, when $n_{\mathrm{RAG}}: n_{\mathrm{HEP}}$ are 4:6, 5: 5 and 6:4, the buffer platform appears, and as the HEP content decreases, the steps gradually become steeper, the amount of $\mathrm{NaOH}$ was reduced from $1.5 \mathrm{~mL}$ to $0.6 \mathrm{~mL}$. The main reason was that the emergence of the platform in the first stage of the acid-base titration curve was the neutralization of $\mathrm{HCl}$ with $\mathrm{NaOH}$, and the platform in the second stage was $-\mathrm{NH}^{+}$deprotonation to form a buffer. Under this system, the buffering capacity of RPUMs was proportional to the $-\mathrm{NH}^{+}$dissociation degree. Therefore, the dissociation capacity and molar concentration of $-\mathrm{NH}^{+}$were the main factors affecting the dissociation degree. Thus, with the amount of HEP decreases, the relative content of hydrophilic segments per unit volume decreases, the molar concentration of $-\mathrm{NH}^{+}$decreases, and the deprotonation process of $-\mathrm{NH}^{+}$gradually overlaps with the neutralization reaction of strong acids and bases in the first stage.

\subsection{Effect of Solvent Types on the Properties of RPUMs}

At the solid content of RPU solution was $30 \%, n_{\mathrm{RAG}}: n_{\mathrm{HEP}}=4: 6$, the effect of solvent mixtures on the performance of RPUMs was investigated. It can be seen from Table 5 and Fig. 6 that the RPUMs prepared by different kinds of solvents are all spherical. There had some polymer fragments and relatively low yields when the $\mathrm{CCl}_{4} /$ toluene and pure toluene are used as solvent. The main reason may be that, $\mathrm{CCl}_{4}$ and toluene are non-polar solvents with low solubility parameters and poor compatibility with the more polar RPU molecular chain. Under the condition, the system displayed a non-uniform two-phase, and resultant RPU exhibited different emulsification degrees in the water phase, leading to the microspheres with large PDI value and poor mono-dispersity.

Table 5: Particle size data of the RPUMs with different kinds of solvent mixture

\begin{tabular}{llllll}
\hline Samples (wt. ratio) & solubility parameter & $\mathrm{D}_{\mathrm{n}} / \mu \mathrm{m}$ & $\mathrm{D}_{w} / \mu \mathrm{m}$ & $\mathrm{PDI}$ & Yield/\% \\
\hline $\mathrm{CCl}_{4} /$ Toluene (13/1) & 8.88 & 26.45 & 40.02 & 1.51 & 82.5 \\
Toluene & 8.90 & 54.10 & 71.20 & 1.32 & 91.7 \\
NBA/Toluene (13/1) & 9.08 & 71.75 & 84.70 & 1.18 & 96.2 \\
DMF/Toluene (13/1) & 9.01 & 65.62 & 78.62 & 1.19 & 96.6 \\
\hline
\end{tabular}

In addition, the boiling point of $\mathrm{CCl}_{4}$ is $76^{\circ} \mathrm{C}$, which is slightly lower than the polymerization temperature $\left(80^{\circ} \mathrm{C}\right)$. The microspheres could be damaged during the polymerization reaction due to $\mathrm{CCl}_{4}$ and toluene dispersed in the spherical cavity, continuously migrated from the inside to the outside, and the yield was relatively low. However, as the polarity of the mixed solvent increases, the solubility parameter increases, thus the compatibility of the solvent and RPU improved. In the case, the RPU can be dissolved in the solvent to form a homogeneous phase, and the vesicles obtained after emulsification are more uniform. As the good solvent was added, the RPU segment was more stretched in the medium, resulting in the looser and swelled latex particle, the microspheres particle size increased.

It can be seen from Fig. 7 and Table 6 that the RPUMs with DMF/toluene mixture as pore-forming agent shows no obvious buffering platform. However, when the pore-forming agent is NBA/toluene, toluene or $\mathrm{CCl}_{4} /$ toluene, the resultant RPUMs all show certain amount of buffering capacity, from 1.3 to $1.6 \mathrm{~mL}$. The results showed that the protonation and deprotonation ability of $-\mathrm{NH}^{+}$of RPUMs synthesized under different kinds of porogens were different. In the process of RPUMs forming, RPU was used as selfemulsifier to wrap the mixed solvent, and also as monomer to solidify the microspheres shell by polymerizing and cross-linking of the double bonds. In the end, the solvent was removed from the microspheres by solvent volatilization to realize the porosity. Therefore, the number of pores in the microspheres directly affects the diffusion of $\mathrm{H}^{+}$in the microspheres, and then affects the amount of the 
protonated tertiary amine. When the pore-forming agent was DMF/toluene, the resultant microspheres were solid, due to the difficult diffusion of $\mathrm{H}^{+}$in the microspheres, resulting in the relatively small amount of $-\mathrm{NH}^{+}$formed by the protonation of tertiary amine, so the microspheres had poor buffering performance. In contrast, when the pore-forming agent was $\mathrm{CCl}_{4}$ /toluene, toluene and NBA/toluene, the resultant microspheres were hollow, and the spherical shell contained a large number of pores with high specific area. After protonation, the concentration of $-\mathrm{NH}^{+}$in the spherical shells was high, and the microspheres had good buffering performance.

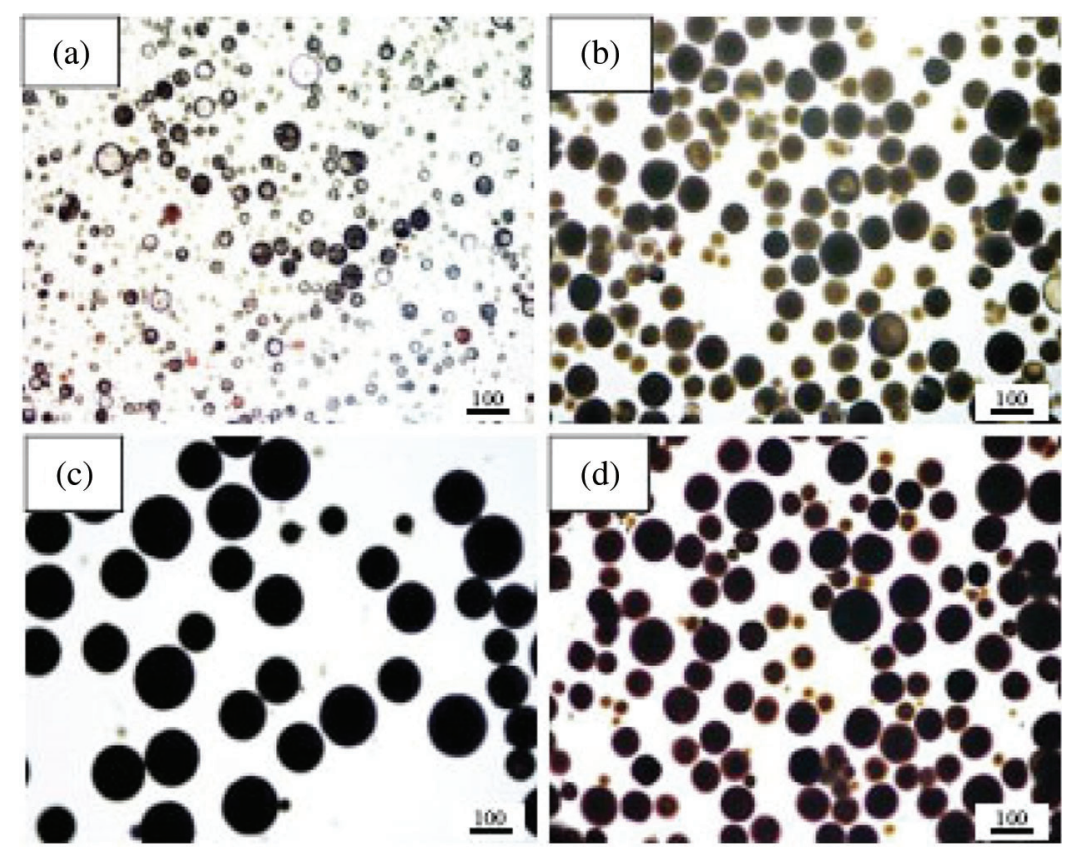

Figure 6: Optical microscope of RPUMs with different solvents (a: $\mathrm{CCl}_{4} /$ Toluene; b: Toluene; c: NBA /Toluene; d: DMF/Toluene)

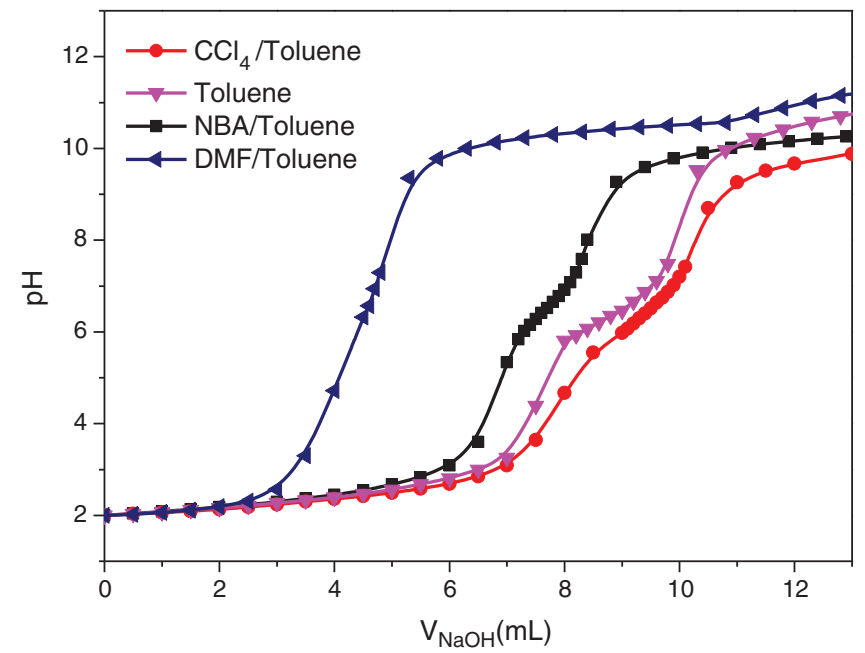

Figure 7: Effect of different kinds of solvent on the pH sensitivity of RPUMs 
Table 6: Dissociative parameters of RPUMs with different kinds of solvents

\begin{tabular}{lllll}
\hline Sample & $\mathrm{CCl}_{4} /$ Toluene & Toluene & $\mathrm{NBA} /$ Toluene & $\mathrm{DMF} /$ Toluene \\
\hline$\Delta \mathrm{V}_{\mathrm{NaOH}} / \mathrm{mL}$ & $1.4 \pm 0.03$ & $1.6 \pm 0.03$ & $1.3 \pm 0.03$ & - \\
$\alpha$ & 0.4826 & 0.4262 & 0.4043 & - \\
\hline
\end{tabular}

\subsection{FTIR Spectrum Analysis}

The infrared spectrum of RPUMs is shown in Fig. 8. The stretching vibration peak at $3387 \mathrm{~cm}^{-1}$ is attributed to $-\mathrm{NH}$, the peaks at 2934 and $2863 \mathrm{~cm}^{-1}$ are attributed to $-\mathrm{CH}_{2}$ and $-\mathrm{CH}_{3}-$, respectively; the peak at $1725 \mathrm{~cm}^{-1}$ is carbonyl $\mathrm{C}=\mathrm{O}$, whereas the peak at $1533 \mathrm{~cm}^{-1}$ is from deformation vibration of secondary amide $\mathrm{N}-\mathrm{H}$. At the same time, the $-\mathrm{CH}_{2}-$ plane vibration absorption peak at $771 \mathrm{~cm}^{-1}$ of the original double bond is enhanced, indicating that RPUMs were successfully prepared by radical polymerization of RPU.

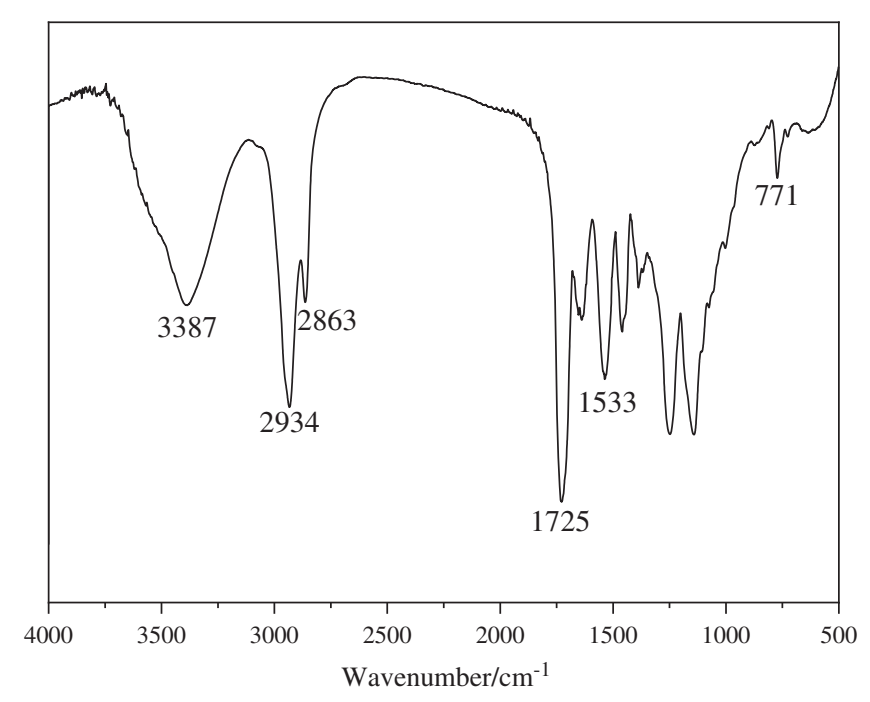

Figure 8: The FTIR spectrum of RPUMs

\subsection{SEM Analysis}

The SEM images of the RPUMs prepared from $\mathrm{CCl}_{4} /$ toluene, toluene, $\mathrm{NBA} /$ toluene and $\mathrm{DMF} /$ toluene as porogens are shown in Fig. 9. It can be seen from Fig. 9 that RPUMs display very clear porous structure. The microspheres prepared with $\mathrm{CCl}_{4} /$ toluene have a larger surface pore diameter and fewer pores than others. From the analysis of the sphere formation mechanism mentioned above, the initial cross-linking reaction was carried out in an $\mathrm{O} / \mathrm{W}$ emulsion, and the hydrophilic chain extender was ordered toward the hydrophilic phase, while the other PU segment was arranged toward the oil phase. As the hydrophobic phase, the solvent was wrapped inside the microspheres, and gradually migrated from inside to outside under heating conditions to form pores. As the boiling point of $\mathrm{CCl}_{4}$ is $76^{\circ} \mathrm{C}$, whereas the polymerization proceeded at $80^{\circ} \mathrm{C}$, thus $\mathrm{CCl}_{4}$ could be quickly moved to outside during the polymerization process. The shells with not fully cured were dissolved or destroyed by volatilization of solvent, thus more lager pore size produced [13]. The higher boiling points of toluene, NBA and DMF led to the lower volatilization rate at the polymerization temperature, and the larger volume of organic solvent enclosed inside the microspheres, as it volatilized gradually, the more compact pores produced. 

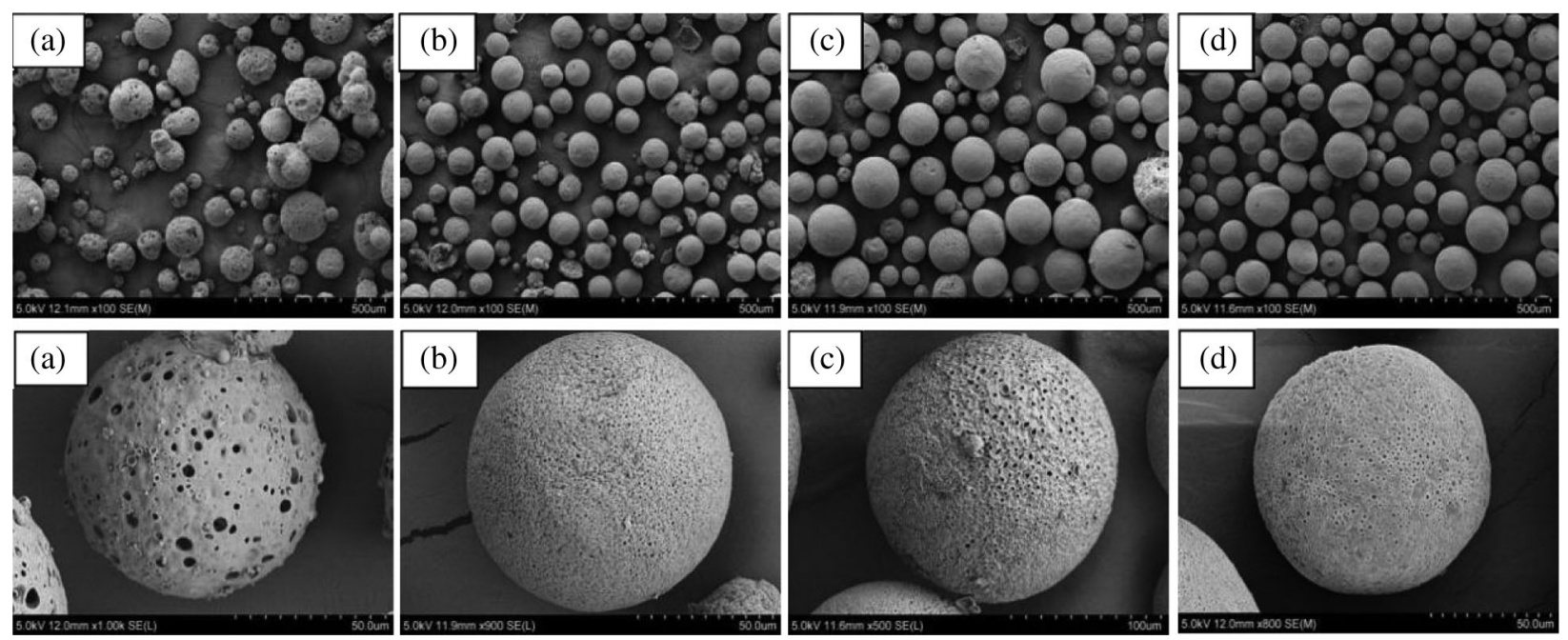

Figure 9: SEM images of RPUMs with different solvents (a: $\mathrm{CCl}_{4} /$ Toluene; b: Toluene; c: NBA/Toluene; d: DMF /Toluene)

\subsection{TEM Analysis}

TEM images of the RPUMs prepared with different kinds of mix-solvents are shown in Fig. 10. When $\mathrm{CCl}_{4} /$ toluene and toluene were used as porogens, the prepared microspheres had good cavity structure with the shell thickness of $\sim 0.2 \mu \mathrm{m}$, while $\mathrm{NBA} /$ toluene and $\mathrm{DMF} /$ toluene were used as porogens, the microspheres were solid, no hollow structure was found. According to the formation mechanism of hollow microspheres, the solvent continuously migrated out from the inside solid phase (polymer phase), the hollow microspheres formed. Non-polar solvents, such as $\mathrm{CCl}_{4}$ and toluene, are not miscible with water, so they were easy to migrate from inside to outside, and hollow structure microspheres could be obtained. Polar solvents, such as BNA and DMF, are miscible with water, and they were not easy to migrate from water and wrapped by the hydrophobic phase, so solid microspheres were obtained.
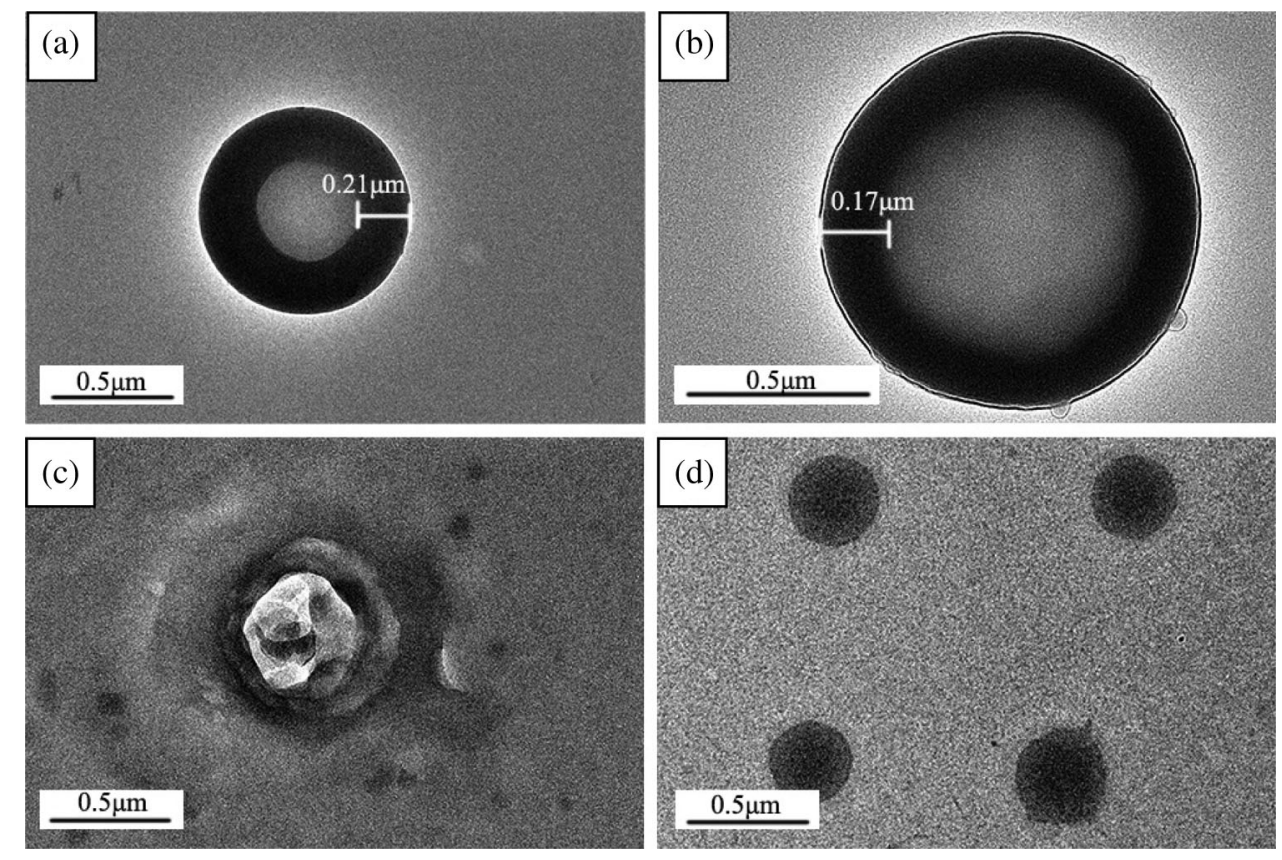

Figure 10: TEM images of RPUMs with different solvents (a: $\mathrm{CCl}_{4} /$ Toluene; b: Toluene; c: NBA/Toluene; d: DMF/Toluene) 


\section{Conclusion}

pH-responsive RPUMs were successfully prepared by self-assembly and self-polymerization methods using rosin-based RPU as both self-emulsifier and polymerizing monomer. The best synthesis conditions for synthetic RPUMs were as follows: $\mathrm{n}_{\mathrm{RAG}}: \mathrm{n}_{\mathrm{HEP}}=4: 6, \mathrm{~m}_{\mathrm{RPU}}: \mathrm{m}_{\text {toluene }}=3: 7$, AIBN level was $1.0 \mathrm{wt} . \%$, emulsification and polymerization mixing speed were $400 \mathrm{r} \cdot \mathrm{min}^{-1}$, the RPUMs synthesized under this condition had porous-hollow structure with a buffer volume of $1.6 \mathrm{~mL}$.

Funding Statement: The authors greatly acknowledge the financial support from the National Natural Science Foundation of China (No. 51863007).

Conflicts of Interest: The authors declare that they have no conflicts of interest to report regarding the present study.

\section{References}

1. Fazal, M. A., Haseeb, A., Masjuki, H. (2011). Biodiesel feasibility study: An evaluation of material compatibility; performance; emission and engine durability. Renewable \& Sustainable Energy Reviews, 15(2), 1314-1324. DOI 10.1016/j.rser.2010.10.004.

2. Wilbon, P. A., Chu, F. X., Tang, C. B. (2013). Progress in renewable polymers from Natural Terpenes, Terpenoids, and Rosin. Macromolecular Rapid Communications, 34(1), 8-37. DOI 10.1002/marc.201200513.

3. Szymon, K., Paula, O., Kornelia, M., Ewa, W. (2019). Advances in rosin-based chemicals: The latest recipes, applications and future trends. Molecules, 24(9), 1651. DOI 10.3390/molecules24091651.

4. Zhang, Y., Hourston, D. J. (1998). Rigid interpenetrating polymer network foams prepared from a rosin-based polyurethane and an epoxy resin. Journal of Applied Polymer Science, 69(2), 271-281. DOI 10.1002/(ISSN) 1097-4628.

5. Zhang, L., Jiang, Y., Xiong, Z., Liu, X., Na, H. et al. (2013). Highly recoverable rosin-based shape memory polyurethanes. Journal of Materials Chemistry A, 1(10), 3263. DOI 10.1039/c3ta01655b.

6. Carbonell-Blasco, P., Martín-Martínez, J. M., Antoniac, I. V. (2013). Synthesis and characterization of polyurethane sealants containing rosin intended for sealing defect in annulus for disc regeneration. International Journal of Adhesion and Adhesives, 42(1), 11-20. DOI 10.1016/j.ijadhadh.2012.11.011.

7. Xu, X., Shang, S., Song, Z., Cui, S., Wang, H. et al. (2011). Preparation and characterization of rosin-based waterborne polyurethane from maleopimaric acid polyester polyol. Bioresources, 6(3), 2460-2470.

8. Howard, G. H. (2002). Biodegradation of Polyurethane: A Review. International Biodeterioration \& Biodegradation, 49(4), 245-252. DOI 10.1016/S0964-8305(02)00051-3.

9. Li, J., Lai, X. Y., Xing, C. J., Wang, D. (2010). One-pot synthesis of porous hematite hollow microspheres and their application in water treatment. Journal of Nanoscience and Nanotechnology, 10(11), 7707-7710. DOI 10.1166/ jnn.2010.2795.

10. Liu, X. H., Jin, X. B., Ma, P. X. (2011). Nanofibrous hollow microspheres self-assembled from star-shaped polymers as injectable cell carriers for knee repair. Nature Materials, 10(5), 398-406. DOI 10.1038/nmat2999.

11. Yang, X. Y., Chen, L., Huang, B., Bai, F., Yang, X. (2009). Synthesis of pH-sensitive hollow polymer microspheres and their application as drug carriers. Polymer, 50(15), 3556-3563. DOI 10.1016/j.polymer.2009.06.027.

12. Shao, J., Yu, C., Bian, F., Zeng, Y., Zhang, F. (2019). Preparation and properties of hydrophilic rosin-based aromatic polyurethane microspheres. ACS Omega, 4(2), 2493-2499. DOI 10.1021/acsomega.8b03334.

13. Pu, W., Fu, D., Xia, H., Wang, Z. (2017). Preparation of hollow polyurethane microspheres with tunable surface structures via electrospraying technology. RSC Advances, 7(79), 49828-49837. DOI 10.1039/C7RA09831F.

14. Fan, J. B., Huang, C., Jiang, L., Wang, S. T. (2013). Nanoporous microspheres: from controllable synthesis to healthcare applications. Journal of Materials Chemistry B, 1(17), 2222. DOI 10.1039/c3tb00021d.

15. Li, M., Harbron, R. L., Weaver, J., Binks, B., Mann, S. (2013). Electrostatically gated membrane permeability in inorganic protocells. Nature Chemistry, 5(6), 529-536. DOI 10.1038/nchem.1644. 
16. Jens, G., Dietmar, A., Linge, W., Giuseppe, G., Brigitte, V. (2012). Synthetic bio-nanoreactor: Mechanical and chemical control of polymersome membrane permeability. Angewandte Chemie International Edition, 51(18), 4448-4451. DOI 10.1002/anie.201108814.

17. Rona, C., Leticia, H., Dirk, V., Shalitha, A., Brigitte, L. et al. (2010). Engineering advanced capsosomes: Maximizing the number of subcompartments, cargo retention, and temperature-triggered reaction. ACS Nano, 4(3), 1351-1361. DOI 10.1021/nn901843j.

18. Yang, T., Wang, Y., Ke, H., Wang, Q., Lv, X. et al. (2016). Protein-nanoreactor-assisted synthesis of semiconductor nanocrystals for efficient cancer theranostics. Advanced Materials, 28(28), 5923-5930. DOI 10.1002/adma.201506119.

19. Lee, Y., Fukushima, S., Bae, Y., Hiki, S., Ishii, T. et al. (2007). A protein nanocarrier from charge-conversion polymer in response to endosomal pH. Journal of the American Chemical Society, 129(17), 5362-5363. DOI 10.1021/ja071090b.

20. Bae, Y., Jang, W. D., Nishiyama, N., Fukushima, S., Kataoka, K. (2005). Multifunctional polymeric micelles with folate-mediated cancer cell targeting and $\mathrm{pH}$-triggered drug releasing properties for active intracellular drug delivery. Molecular BioSystems, 1(3), 242. DOI 10.1039/b500266d.

21. Santosh, A., Hu, C. M., Zhang, L. F. (2010). Polymer-cisplatin conjugate nanoparticles for acid-responsive drug delivery. ACS Nano, 4(1), 251-258. DOI 10.1021/nn9014032.

22. Wang, A., Gao, H., Sun, Y. F., Sun, Y. L., Yang, Y. et al. (2013). Temperature- and pH-responsive nanoparticles of biocompatible polyurethanes for doxorubicin delivery. International Journal of Pharmaceutics, 441(1-2), 30-39. DOI 10.1016/j.ijpharm.2012.12.021.

23. Filippov, S., Hrubý, M., Čestmír, K., Mackova, H., Spirkova, M. et al. (2008). Novel pH-responsive nanoparticles. Langmuir: The ACS Journal of Surfaces and Colloids, 24(17), 9295-9301. DOI 10.1021/la801472x. 